\title{
René Descartes
}

\section{Correspondance}

\author{
Un document produit en version numérique par Denis Collin, bénévole, \\ docteur ès lettres et sciences humaines, \\ Professeur agrégé de philosophie, au Lycée Aristide Briand à Évreux (Eure), \\ Courriel : denis.collin@wanadoo.fr \\ Site web : http://perso.wanadoo.fr/denis.collin
}

\section{Dans le cadre de la collection: "Les classiques des sciences sociales" dirigée et fondée par Jean-Marie Tremblay, professeur de sociologie au Cégep de Chicoutimi}

Site web: http://www.uqac.uquebec.ca/zone30/Classiques_des_sciences_sociales/index.html

Une collection développée en collaboration avec la Bibliothèque Paul-Émile-Boulet de l'Université du Québec à Chicoutimi Site web: http://bibliotheque.uqac.uquebec.ca/index.htm 
Un document produit en version numérique par Denis Collin, bénévole, docteur ès lettres et sciences humaines,

Professeur agrégé de philosophie, au Lycée Aristide Briand à Évreux (Eure),

Courriel : denis.collin@,wanadoo.fr

Site web : $\underline{\text { http://perso.wanadoo.fr/denis.collin }}$

à partir de :

\section{René Descartes, 1596-1650}

Correspondance (éditée de 1657 à 1659)

Une édition électronique réalisée à partir de la correspondance de René DESCARTES (éditée entre 1657 et 1659), Oeuvres de Descartes. Édition Adam et Paul Tanney, édition de référence publiée pour la première fois lors du tricentenaire de Descartes (1896). L'édition AT a été reproduite en fac-similé par Vrin en 1996 (pour le quatrième centenaire). Tome I : Correspondance Avril 1622- février 1638; Tome II : mars 1638 - décembre 1639; Tome III: janvier 1640 - juin 1643; Tome IV: juillet 1643 - avril 1647; Tome V: mai 1646 - février 1650 .

Polices de caractères utilisée :

Pour le texte: Times, 12 points.

Pour les citations : Times 10 points.

Pour les notes de bas de page : Times, 10 points.

Édition électronique réalisée avec le traitement de textes Microsoft Word 2000.

Mise en page sur papier format

LETTRE (US letter), 8.5" x 11"')

Édition complétée le 18 décembre 2002 à Chicoutimi, Québec.

\section{Fait avec}

Macintosh 


\section{Table des matières}

1- La lettre de Descartes à Christine de Suède du 20 novembre 1674 sur la conciliation des éthiques stoïciennes et épicuriennes.

(AT. V - pp. 81-86)

[Version française de Clerselier, tome I, lettre i, pp. 1-5.]

2- $\quad$ La lettre de Descartes à Élisabeth (21 mai 1643)

(AT. III, pp. 663-668)

[Version française de Clerselier : Tome I, lettre 29, p.89-92]

3- $\quad$ La lettre de Descartes à Mersenne (27 mai 1641 ?)

AT. III, pp.378-382

[Version française de Clerselier, tome 1, lettre 112, pp. 506-509]

4- $\quad$ La lettre de Descartes au Marquis de Newcastle (sur le langage). (23 novembre 1646)

AT. IV. pp. 568-576

[Version française de Clerselier : tome I, lettre 54, pp. 157-163]

5- $\quad$ Lettre de Descartes à Élisabeth (Egmond, 6 juin 1647.) (AT. IV - pp. 280-287)

[Copie manuscrit Marburg, Staatarchiv, lettre de Descartes n4]

6- $\quad$ Lettre de Descartes à Chanut (6 juin 1647.)

(AT. V - pp. 50-58)

[Édition Clerselier tome I, lettre 36 pp.119-126]

7- $\quad$ Lettre de Descartes à Gibieuf (19 janvier 1642.)

(AT. II - pp. 472-480)

[Version française de Clerselier, tome 1, lettre 105, p.479-484] 
1-

Lettre de Descartes à

Christine de Suède

\section{Egmond, 20 novembre 1647.}

\section{(AT. V - pp. 81-86) \\ [Version française de Clerselier, tome I, lettre i, pp. 1-5.]}

(La lettre de Descartes à Christine de Suède du 20 novembre 1674 sur la conciliation des éthiques stoïciennes et épicuriennes [DC]).

$\underline{\text { Retour à la table des matières }}$

\section{Madame,}

J'ai appris de Monsieur Chanut qu'il plaît à votre Majesté que j'ai l'honneur de lui exposer l'opinion que j'ai touchant le Souverain Bien, considéré au sens que les philosophes anciens en ont parlé ; et je tiens ce commandement pour une si grande faveur, que le désir que j'ai d'obéir me détourne de toute autre pensée, et fait que, sans excuser mon insuffisance, je mettrai ici en peu de mots tout ce que je pourrai savoir sur cette matière.

On peut considérer la bonté de chaque chose en elle-même, sans la rapporter à autrui, auquel sens il est évident que c'est Dieu qui est le souverain bien, parce qu'il est incomparablement plus parfait que les créatures ; mais on peut 
aussi la rapporter à nous, en ce sens que je ne vois rien que nous devions estimer bien, sinon ce qui nous appartient en quelque façon, et qui est tel que c'est perfection pour nous de l'avoir. Ainsi les philosophes anciens, qui, n'étant point éclairés de la lumière de la Foi, ne savaient rien de la béatitude surnaturelle, ne considéraient que les biens que nous pouvons posséder en cette vie ; et c'était entre ceux-là qu'ils cherchaient lequel était le souverain, c'est-à-dire le principal et le plus grand.

Mais, afin que je le puisse déterminer, je considère que nous ne devons estimer biens, à notre égard, que ceux que nous possédons ou que nous avons le pouvoir d'acquérir. Et cela posé, il me semble que le souverain bien de tous les hommes ensemble est un amas ou un assemblage de tous les biens, tant de l'âme que du corps et de la fortune, qui peuvent être en quelques hommes ; mais que celui d'un chacun en particulier est toute autre chose, et qu'il ne consiste qu'en une ferme volonté de bien faire, et au contentement qu'elle produit. Dont la raison est que je ne remarque aucun autre bien qui me semble si grand ni qui soit entièrement au pouvoir de chacun. Car, pour les biens du corps et de la fortune, ils ne dépendent point absolument de nous; et ceux de l'âme se rapportent tous à deux chefs, qui sont, l'un de connaître, l'autre de vouloir ce qui est bon ; mais la connaissance est souvent au delà de nos forces ; c'est pourquoi il ne reste que notre volonté, dont nous puissions absolument disposer. Et je ne vois point qu'il soit possible d'en disposer mieux, que si l'on a toujours une ferme et constante résolution de faire exactement toutes les choses que l'on jugera être les meilleures et d'employer toutes les forces de son esprit à les bien connaître. Et c'est en cela seul que consistent toutes les vertus ; c'est cela seul qui, à proprement parler, mérite de la louange et de la gloire ; enfin, c'est de cela seul que résulte toujours le plus grand et le plus solide contentement de la vie. Ainsi, j'estime que c'est en cela que consiste le souverain bien.

Et par ce moyen, je pense accorder les deux plus contraires et plus célèbres opinions des anciens, à savoir celle de Zénon, qui l'a mis en la vertu ou en l'honneur, et celle d'Epicure, qui l'a mis au contentement auquel il a donné le nom de volupté. Car, comme tous les vices ne viennent que de l'incertitude et de la faiblesse qui suit l'ignorance, et qui fait naître les repentirs ; ainsi la vertu ne consiste qu'en la résolution et la vigueur avec laquelle on se porte à faire les choses qu'on croit être bonnes, pourvu que cette vigueur ne vienne pas de l'opiniâtreté, mais de ce qu'on sait les avoir autant examinées, qu'on en a moralement le pouvoir. Et bien que ce qu'on fait alors puisse être mauvais, on est assuré néanmoins qu'on fait son devoir; au lieu que si on exécute quelque action de vertu et que cependant on pense mal faire, ou bien qu'on néglige de savoir ce qu'il en est, on n'agit pas en homme vertueux. Pour ce 
qui est de l'honneur et de la louange, on les attribue souvent aux autres biens de la fortune, mais, parce que je m'assure que votre Majesté fait plus de sa vertu que de sa couronne, je ne craindrai point ici de dire qu'il ne me semble pas qu'il y ait rien que cette vertu qu'on ait juste raison de louer. Tous les autres méritent seulement d'être estimés, et non point d'être honorés ou loués, si ce n'est en tant qu'on présuppose qu'ils sont acquis ou obtenus de Dieu par le bon usage du libre arbitre. Car l'honneur et la louange est une espèce de récompense, et il n'y a rien que ce qui dépend de la volonté qu'on ait sujet de récompenser ou de punir.

Il me reste encore ici à prouver que c'est de ce bon usage du libre arbitre, que vient le plus grand et le plus solide contentement de la vie ; ce qui me semble n'être pas difficile, parce que, considérant avec soin en quoi consiste la volupté ou le plaisir, et généralement toutes les sortes de contentement qu'on peut avoir, je remarque, en premier lieu, qu'il n'y en a aucun qui ne soit entièrement en l'âme, bien que plusieurs dépendent du corps ; de même que c'est aussi l'âme qui voit, bien que ce soit par l'entremise des yeux. Puis je remarque qui puisse donner du contentement à l'âme, sinon l'opinion qu'elle a de posséder quelque bien, et que souvent cette opinion n'est en elle qu' une représentation fort confuse, et même que son union avec le corps est cause qu'elle se représente ordinairement certains biens incomparablement plus grands qu'ils ne sont; mais que si elle connaissait distinctement leur juste valeur, son contentement serait toujours proportionné à la grandeur du bien dont il procéderait. Je remarque aussi que la grandeur d'un bien, à notre égard, ne doit pas seulement être mesurée par la valeur de la chose en quoi il consiste, mais principalement aussi par la façon dont il se rapporte à nous ; et qu'outre que le libre arbitre est de soi la chose la plus noble qui puisse être en nous, d'autant qu'il nous rend en quelque façon pareils à Dieu et semble nous exempter de lui être sujets, et que, par conséquent, son bon usage est le plus grand de tous les biens, il est aussi celui qui est le plus proprement nôtre et qui nous importe le plus, d'où il suit que ce n'est que de lui que nos plus grands contentements peuvent procéder. Aussi voit-on, par exemple, que le repos d'esprit et la satisfaction intérieure que ressentent en eux-mêmes ceux qui savent qu'ils ne manquent jamais à faire leur mieux, tant pour connaître le bien que pour l'acquérir, est un plaisir sans comparaison, plus doux, plus durable et plus solide que tous ceux qui viennent d'ailleurs.

J'omets encore ici beaucoup d'autres choses, parce que, me représentant le nombre des affaires qui se rencontrent en la conduite d'un grand Royaume, et dont votre Majesté prend elle-même les soins, je n'ose lui demander plus longue audience. Mais j'envoie à Monsieur Chanut quelques écrits, où j'ai mis mes sentiments plus au long touchant la même matière, afin que, s'il plaît à 
votre Majesté de les voir, il m'oblige de les lui présenter, et que cela aide à témoigner avec combien de zèle et de dévotion, je suis,

Madame,

De votre Majesté,

Le très humble et très obéissant serviteur, Descartes. 
2-

Lettre de Descartes à Élisabeth

\section{Egmond du Hoef, 21 mai 1643}

\section{(AT-III, pp. 663-668) \\ [Version française de Clerselier. Tome I, lettre 29, p.89-92]}

$\underline{\text { Retour à la table des matières }}$

Madame,

La faveur dont votre Altesse m'a honoré, en me faisant recevoir ses commandements par écrit, est plus grande que je n'eusse osé l'espérer ; et elle soulage mieux mes défauts que celle que j'avais souhaitée avec passion, qui était de les recevoir de bouche, si j'eusse pu être admis à l'honneur de vous faire la révérence et de vous offrir mes très humbles services lors que j'étais dernièrement à La Haye. Car j'aurais eu trop de merveilles à admirer en même temps ; et voyant sortir des discours plus qu'humains d'un corps si semblable à ceux que les peintres donnent aux anges, j'eusse été ravi de même façon que me semblent le devoir être ceux qui venant de la terre, entrent nouvellement dans le ciel. Ce qui m'eût rendu moins capable de répondre à votre Altesse, qui sans doute a déjà remarqué en moi ce défaut, lorsque j'ai eu, ci-devant, l'honneur de lui parler ; et votre clémence l'a voulu soulager, en me laissant les traces de vos pensées sur un papier, où, les relisant plusieurs fois, et m'accoutumant à les considérer, j'en suis véritablement moins ébloui, mais je 
n'en ai que d'autant plus d'admiration, remarquant qu'elles ne paraissent pas seulement ingénieuses à l'abord, mais d'autant plus judicieuses et solides qu'on les examine.

Et je puis dire, avec vérité que la question que votre Altesse propose, me semble être celle qu'on peut me demander avec le plus de raison en suite des écrits que j'ai publiés. Car, y ayant deux choses en l'âme humaine, desquelles dépend tout ce que nous pouvons savoir de sa nature, l'une desquelles est qu'elle pense, l'autre qu'étant unie au corps, elle peut agir et pâtir avec lui ; je n'ai quasi rien dit de cette dernière, et me suis seulement étudié à faire bien entendre la première à cause que mon principal dessein était de prouver la distinction qui est entre l'âme et le corps ; à quoi celle-ci seulement a pu servir, et l'autre y aurait été nuisible. Mais parce que votre Altesse voit si clair, qu'on ne lui peut dissimuler aucune chose, je tâcherai ici d'expliquer la façon dont je conçois l'union de l'âme et du corps, et comment elle a la force de le mouvoir.

Premièrement, je considère qu'il y a en nous certaines notions primitives, qui sont comme des originaux, sur le patron desquels nous formons toutes nos autres connaissances. Et il n'y a que fort peu de telles notions ; car, après les plus générales de l'être, du nombre, de la durée, etc., qui conviennent à tout ce que nous pouvons concevoir, nous n'avons pour le corps en particulier, que la notion de l'extension, de laquelle suivent celles de la figure et du mouvement; et pour l'âme seule, nous n'avons que celle de la pensée en laquelle sont comprises les perceptions de l'entendement et les inclinations de la volonté ; enfin, pour l'âme et le corps ensemble, nous n'avons que celle de leur union, de laquelle dépend celle de la force qu'a l'âme de mouvoir le corps, et le corps d'agir sur l'âme, en causant ses sentiments et ses passions.

Je considère aussi que toute la science des hommes ne consiste qu'à bien distinguer ces notions, et à n'attribuer chacune d'elles qu'aux choses auxquelles elles appartiennent. Car, lorsque nous voulons expliquer quelque difficulté par le moyen d'une notion qui ne lui appartient pas, nous ne pouvons manquer de nous méprendre ; comme aussi lorsque nous voulons expliquer une de ces notions par une autre ; car, étant primitives, chacune d'elles ne peut être entendue que par elle-même. Et d'autant que l'usage des sens nous a rendu les notions de l'extension, des figures, et des mouvements beaucoup plus familières que les autres, la principale cause de nos erreurs est que nous voulons ordinairement nous servir de ces notions pour expliquer des choses à qui elles n'appartiennent pas, comme lorsqu' on veut se servir de l'imagination pour concevoir la nature de l'âme, ou bien lorsqu'on veut concevoir la façon dont l'âme meut le corps, par celle dont un corps est mu par un autre corps. 
C'est pourquoi, puisque dans les Méditations que votre Altesse a daigné lire, j'ai tâché de faire concevoir les notions qui appartiennent à l'âme seule, les distinguant de celles qui appartiennent au corps seul, la première chose que je dois expliquer ensuite, est la façon de concevoir celles qui appartiennent à l'union de l'âme et du corps, sans celles qui appartiennent au corps seul ou à l'âme seule. À quoi il me semble que peut servir ce que j'ai écrit à la fin de ma réponse aux sixièmes objections; car nous ne pouvons chercher ces notions simples ailleurs qu'en notre âme, qui les a toutes en soi par sa nature, mais qui ne les distingue pas toujours assez les unes des autres, ou bien les attribue pas aux objets auxquels elle doit les attribuer.

Ainsi je crois que nous avons ci-devant confondu la notion de la force dont l'âme agit dans le corps, avec celle dont un corps agit dans un autre; et que nous avons attribué l'une et l'autre, non pas à l'âme, car nous ne la connaissions pas encore, mais aux diverses qualités des corps, comme à la pesanteur, à la chaleur, et autres, que nous avons imaginé être réelles, c'est-àdire avoir une existence distincte du corps, et, par conséquent, être des substances bien que nous les ayons nommées des qualités. Et nous nous serons servis pour les concevoir, tantôt des notions qui sont en nous pour connaître le corps et tantôt de celles qui y sont pour connaître l'âme, selon que ce que nous leur avons attribué a été matériel ou immatériel. Par exemple, en supposant que la pesanteur est une qualité réelle dont n'avons point d'autre connaissance, sinon qu'elle a la force de mouvoir le corps, dans lequel elle est, vers le centre de la terre, nous n'avons pas de peine à concevoir comment elle meut ce corps, ni comment elle lui est jointe ; et nous ne pensons point que cela se fasse par un attouchement réel d'une superficie contre une autre, car nous expérimentons en nous-mêmes que nous avons une notion particulière pour concevoir cela ; et je crois que nous usons mal de cette notion, en l'appliquant à la pesanteur, qui n'est rien de réellement distingué des corps, comme j'espère montrer en la Physique, mais qu'elle nous a été donnée pour concevoir la façon dont l'âme meut le corps.

Je témoignerais ne pas assez connaître l'incomparable esprit de votre Altesse, si j'employais d'avantage de paroles à m'expliquer, et je serais trop présomptueux, si j'osais penser que ma réponse la doive entièrement satisfaire ; mais je tâcherai d'éviter l'un et l'autre en n'ajoutant rien ici de plus, sinon que, si je suis capable d'écrire ou de dire quelque chose qui lui puisse agréer, je tiendrai toujours à très grande faveur de prendre la plume ou d'aller à La Haye pour ce sujet, et qu'il n'y a rien au monde qui me soit aussi cher que de pouvoir obéir à ses commandements. Mais je ne puis ici trouver place 
à l'observation du serment d'Hippocrate ${ }^{1}$ qu'elle m'enjoint, puisqu'elle ne m'a rien communiqué, qui ne mérite d'être vu et admiré de tous les hommes. Seulement, puis-je dire sur ce sujet, qu'estimant infiniment la vôtre que j'ai reçue, j'en userai comme les avares font de leurs trésors, lesquels ils cachent d'autant plus qu'ils les estiment, et en enviant la vue au reste du monde, ils mettent leur souverain contentement à les regarder. Ainsi je serai bien aise de jouir seul du bien de la voir ; et ma plus grande ambition est de me pouvoir dire, et d'être véritablement, etc.

1 Allusion à la demande d'Élisabeth que Descartes ne rende pas publiques les réponses (cf. lettre Élisabeth à Descartes, 6/16 mai 1643 - AT-III, pp. 660-662 
3-

Lettre de Descartes à

Mersenne

\title{
Egmond du Hoef, 27 mai 1641 ?
}

\author{
AT. III, pp.378-382 \\ [Version française de Clerselier, tome 1, lettre 112, pp. 506- \\ 509]
}

$\underline{\text { Retour à la table des matières }}$

Pour le libre arbitre, je suis entièrement d'accord avec le R.P ${ }^{1}$. Et pour expliquer encore plus nettement mon opinion, je désire, premièrement, que l'on remarque que l'Indifférence me semble signifier proprement cet état dans lequel la volonté se trouve lors qu'elle n'est point portée, par la connaissance de ce qui est vrai ou de ce qui est bon à suivre un parti plutôt que l'autre; et c'est en ce sens que je l'ai prise lorsque j'ai dit que le plus bas degré de la liberté consistait à se pouvoir déterminer aux choses auxquelles nous sommes tout à fait indifférents. Mais peut-être que, par ce mot d'Indifférence, il y en a d'autres qui entendent qui entendent cette faculté positive que nous avons de nous déterminer à l'un ou à l'autre des deux contraires, c'est-à-dire à poursuivre ou à fuir, à affirmer ou à nier un même chose. Sur quoi j'ai à dire que je n'ai jamais nié que cette faculté positive se trouvât en la volonté ; tant s'en

1 Révérend Père Mersenne. 
faut, j'estime qu'elle s'y rencontre, non seulement toutes les fois qu'elle se détermine à ces fortes actions, où elle n'est point emportée par le poids d'aucune raison vers un côté plutôt que vers un autre ; mais même qu'elle se trouve mêlée dans toutes les autres actions en sorte qu'elle ne se détermine jamais qu'elle ne la mette en usage ; jusque là que, lors même qu'une raison fort évidente nous porte à une chose, quoique moralement parlant, il soit difficile que nous puissions faire le contraire, parlant néanmoins absolument, nous le pouvons ; car il nous est toujours libre de nous empêcher de poursuivre un bien qui nous est clairement connu, ou d'admettre une vérité évidente, pourvu seulement que nous pensions que c'est un bien de témoigner par là la liberté de notre franc-arbitre.

De plus, il faut remarquer que la liberté peut être considérée dans les actions de la volonté, ou avant qu'elles soient exercées, ou au moment même qu'on les exerce.

Or il est certain qu'étant considérée dans les actions de la volonté avant qu'elle soient exercées, elle emporte avec soi l'Indifférence, prise dans le second sens que je la viens d'expliquer, et non point dans le premier. C'est-àdire qu'avant que notre liberté se soit déterminée, elle est toujours libre, ou a la puissance de choisir l'un ou l'autre des deux contraires, mais elle n'est pas toujours indifférente ; au contraire, nous ne délibérons jamais qu'à dessein de nous ôter de cet état, où nous ne savons quel parti prendre, ou pour nous empêcher d'y tomber. Et bien qu'opposant notre propre jugement aux commandements des autres, nous ayons coutume de dire que nous sommes plus libres à faire des choses dont il ne nous est rien commandé, et où il nous est permis de suivre notre jugement, qu'à faire celles qui nous sont commandées ou défendues ; toutefois, en opposant nos jugements ou nos connaissances les unes aux autres, nous ne pouvons pas ainsi dire que nous soyons plus libres à faire des choses qui ne nous semblent ni bonnes ni mauvaises, ou dans lesquelles nous voyons autant de bien que de mal, qu'à faire celles où nous apercevons beaucoup plus de bien que de mal. Car la grandeur de la liberté consiste, ou dans la grande facilité que l'on a à se déterminer, ou dans le grand usage de cette puissance positive que nous avons de suivre le pire, encore que nous connaissions le meilleur. Or est-il que, si nous embrassons les choses que notre raison nous persuade être bonnes, nous nous déterminons alors avec beaucoup de facilité ; que si nous faisons le contraire, nous faisons alors un plus grand usage de cette puissance positive ; et ainsi nous pouvons toujours agir avec plus de liberté touchant les choses où nous voyons plus de bien que de mal, que touchant celles que nous appelons Indifférentes. Et en ce sens-là aussi, il est vrai de dire que nous faisons moins librement les choses qui nous sont commandées, et auxquelles, sans cela, nous ne nous porterions jamais de 
nous-mêmes, que nous ne faisons celles qui ne nous sont point commandées. D'autant que le jugement qui nous fait croire que ces choses-là sont difficiles, s'oppose à celui qui nous dit qu'il est bon de faire ce qui nous est commandé ; lesquels deux jugements d'autant plus également nous meuvent, et plus mettent-ils en nous de cette indifférence, prise dans le sens que j'ai le premier expliqué, c'est-à-dire qui met la volonté dans un état à ne savoir à quoi se déterminer.

Maintenant, la liberté étant considérée dans les actions de la volonté, au moment même qu'elles sont exercées, alors elle ne contient aucune indifférence, en quelque sens qu' on la veuille prendre ; parce que ce qui se fait, ne pas ne point se faire dans le même qu'il se fait ; mais elle consiste seulement dans la facilité qu'on a d'opérer, laquelle, à mesure qu'elle croît, à mesure aussi la liberté augmente ; et alors faire librement une chose, ou la faire volontiers ou bien la faire volontairement, ne sont qu'une même chose. Et c'est en ce sens-là que j'ai écrit que je me portais d'autant plus librement à une chose, que j'y étais poussé par plus de raisons, parce qu'il est certain que notre volonté se meut alors plus facilement et avec plus d'impétuosité. 
4-

\title{
Descartes : Lettre au
} marquis de Newcastle

\section{3 novembre 1646}

\author{
AT. IV. pp. 568-576 \\ [Version française de Clerselier: tome I, lettre 54, pp. 157-163]
}

$\underline{\text { Retour à la table des matières }}$

\section{MONSEIGNEUR,}

Les faveurs que je reçois par les lettres qu'il a plu à votre excellence de m'écrire, et les marques qu'elles contiennent d'un esprit qui donne plus de lustre à la haute naissance qu'il n'en reçoit d'elle, m'obligent de les estimer extrêmement; mais il semble, outre cela, que la fortune veuille montrer qu'elle les met au rang des plus grands biens que je puis posséder, parce qu'elle les arrête par les chemins et ne permet pas que je les reçoive, qu'après avoir fait tous ses efforts pour l'empêcher. Ainsi, j'eus l'honneur d'en recevoir une l'année passée qui avait été quatre mois à venir de Paris ici ; et celle que je reçois maintenant est du cinquième Janvier ; mais parce que Monsieur de B. m'assure que vous avez déjà été averti du retardement, je ne m'excuse point de n'y avoir pas plutôt fait réponse. Et d'autant que les choses dont il vous a plu m'écrire sont seulement des considérations touchant les sciences, qui ne dépendent point des changements du temps ni de la fortune, 
j'espère que ce que j'y pourrai maintenant répondre, ne vous sera pas moins agréable que si vous l'aviez reçu il y a dix mois.

Je souscris en tout au jugement que votre Excellence fait des chimistes, et crois qu'ils ne font que dire des choses hors de l'usage commun pour faire semblant de savoir ce qu'ils ignorent. Je crois aussi que ce qu'ils disent de la résurrection des fleurs par leur sel, n'est qu'une imagination sans fondement, et que leurs extraits ont d'autres vertus que celles des plantes dont ils sont tirés. Ce qu'on expérimente bien clairement, en ce que le vin, le vinaigre et l'eau de vie, qui sont trois extraits qu'on peut faire des mêmes raisins, ont des goûts et des vertus si diverses. Enfin, selon mon opinion, leur sel, leur soufre et leur mercure ne diffèrent pas plus entre eux que les quatre Éléments des Philosophes, ni guère plus que l'eau diffère de la glace, de l'écume et de la neige ; car je pense que tous les corps sont faits d'une même matière, et qu'il n'y a rien qui fasse la diversité entre eux, sinon que les petites parties de cette matière qui composent les uns, ont d'autres figures, ou sont arrangées autrement, que celles qui composent les autres. Ce que j'espère que votre Excellence pourra voir bientôt expliqué assez au long en mes Principes de Philosophie, qu'on va imprimer en français.

Je ne sais rien de particulier touchant la génération des pierres, sinon que je les distingue des métaux, en ce que les petites parties qui composent les métaux sont notablement plus grosses que les leurs ; et je les distingue des os, des bois durs et autres parties des animaux et végétaux, en ce qu'elles ne croissent pas comme eux, comme eux, par le moyen de quelque suc qui coule par de petits canaux en tous les endroits de leur corps, mais seulement par l'addition de quelques parties qui s'attachent à elles par dehors, ou bien s'engagent au dedans de leurs pores. Ainsi, je ne m'étonne point de ce qu'il y a des fontaines où il s'engendre des cailloux : car je crois que l'eau de ces fontaines entraîne avec soi de petites parties des rochers par où elle passe, lesquelles sont de telles figures qu'elles s'attachent facilement les unes aux autres, lorsqu'elles viennent à se rencontrer, et que l'eau qui les amène, étant moins vive et moins agitée qu'elle n'a été dans les veines de ces rochers, les laisse tomber; et il en est quasi de même de celles qui s'engendrent dans le corps des hommes. Je ne m'étonne pas aussi de la façon dont la brique se fait ; car je crois que sa dureté vient de ce que, l'action du feu faisant sortir d'entre ses parties, non seulement les parties de l'eau, que j'imagine longues et glissantes, ainsi que de petites anguilles, qui coulent dans les pores des autres corps sans s'y attacher, et auxquelles seules consiste l'humidité ou la moiteur de ces corps, comme je l'ai dit dans les Météores, mais aussi toutes les autres parties de leur matière, qui ne sont pas bien dures et bien fermes, au moyen de quoi celles qui demeurent se joignent plus étroitement l'une à l'autre, et ainsi 
font que la brique est plus dure que l'argile, bien qu'elle ait des pores plus grands, dans lesquels il entre par après d'autres parties d'eau ou d'air, qui la peuvent rendre avec cela plus pesante.

Pour la nature de l'argent vif, je n'ai pas encore fait toutes les expériences dont j'ai besoin pour la connaître exactement ; mais je crois néanmoins pouvoir assurer que ce qui le rend si fluide qu'il est, c'est que les petites parties dont il est composé, sont si unies et si glissantes qu'elles ne se peuvent aucunement attacher l'une à l'autre, et qu'étant plus grosses que celles de l'eau, elles ne donnent guère de passage parmi elles à la matière subtile que j'ai nommée le second élément, mais seulement à celle qui est très subtile, et que j'ai nommée le premier élément. Ce qui me semble suffire pour pouvoir rendre raison de toutes celles de ces propriétés qui m'ont été connues jusqu'ici : car c'est l'absence de cette matière du second élément qui l'empêche d'être transparent, et qui le rend fort froid ; c'est l'activité du premier élément, avec la disproportion qui est entre ses parties et celles de l'air ou des autres corps, qui fait que ses petites gouttes se relèvent plus en rond sur une table, que celles de l'eau; et c'est aussi la même disproportion, qui est cause qu'il ne s'attache point à nos mains comme l'eau, qui a donné sujet de penser qu'il n'est pas humide comme elle ; mais il s'attache bien au plomb et à l'or, c'est pourquoi on peut dire à leur égard qu'il est humide.

J'ai bien du regret de ne pouvoir lire le livre de M. d'Igby, faute d'entendre l'anglais ; je m'en suis fait interpréter quelque chose ; et parce que je suis entièrement disposé à obéir à la raison, et que je sais que son esprit est excellent, j'oserais espérer, si j'avais l'honneur de conférer avec lui, que mes opinions s'accorderaient aisément avec les siennes.

Pour ce qui est de l'entendement ou de la pensée que Montaigne et quelques autres attribuent aux bêtes, je ne puis être de leur avis. Ce n'est pas que je m'arrête à ce qu'on dit, que les hommes ont un empire absolu sur tous les autres animaux ; car j'avoue qu'il y en a de plus forts que nous, et crois qu'il y en peut aussi avoir qui aient des ruses naturelles, capables de tromper les hommes les plus fins. Mais je considère qu'ils ne nous imitent ou surpassent qu'en celles de nos actions qui ne sont point conduites par notre pensée ; car il arrive souvent que nous marchons ou mangeons, sans penser en aucune façon à ce que nous faisons; et c'est tellement sans user de notre raison que nous repoussons les choses qui nous nuisent, et parons les coups que l'on nous porte, qu'encore que nous voulussions expressément ne point mettre nos mains devant notre tête, lorsqu'il arrive que nous tombons, nous ne pourrions nous en empêcher. Je crois aussi que nous mangerions, comme les bêtes, sans l'avoir appris, si nous n'avions aucune pensée; et l'on dit que ceux qui 
marchent en dormant, passent quelquefois des rivières à la nage, où ils se noieraient, étant éveillés. Pour les mouvements de nos passions, bien qu'ils soient accompagnés en nous de pensées, à cause que nous avons la faculté de penser, il est néanmoins très évident qu'ils ne dépendent pas d'elle, parce qu'ils se font souvent malgré nous, et que, par conséquent, ils peuvent être dans les bêtes, et même plus violents qu'ils ne sont dans les hommes, sans qu'on puisse pour cela conclure qu'elles ont des pensées.

Enfin, il n'y aucune de nos actions extérieures, qui puisse assurer ceux qui les examinent, que notre corps n'est pas seulement une machine qui se remue de soi-même, mais qu'il y a aussi en lui une âme qui a des pensées, excepté les paroles, ou autres signes faits à propos des sujets qui se présentent, sans se rapporter à aucune passion. Je dis les paroles ou autres signes parce que les muets se servent de signes en même façon que nous de la voix ; et que ces signes soient à propos, pour exclure le parler des perroquets, sans exclure celui des fous, qui ne laisse pas d'être à propos des sujets qui se présentent, bien qu'il ne suive pas la raison; et j'ajoute que ces paroles ou signes ne se doivent rapporter à aucune passion, pour exclure non seulement les cris de joie ou de tristesse, et semblables, mais aussi tout ce qui peut être enseigné par artifice aux animaux ; car si on apprend à une pie à dire bonjour à sa maîtresse, lorsqu'elle la voit arriver, ce ne peut être qu'en faisant que la prolation de cette parole devienne le mouvement de quelqu'une de ses passions ; à savoir, ce sera un mouvement de l'espérance qu'elle a de manger, si l'on a toujours accoutumé de lui donner quelque friandise, lorsqu'elle l'a dit; et ainsi toutes les choses qu'on fait faire aux chiens, aux chevaux et aux singes, ne sont que les mouvements de leur crainte, de leur espérance, de leur joie, en sorte qu'ils les peuvent faire sans aucune pensée. Or, il est, ce me semble, fort remarquable que la parole ainsi définie, ne convient qu'à l'homme seul. Car, bien que Montaigne et Charron aient dit qu'il y a plus de différence d'homme à homme que d'homme à bête, il ne s'est toutefois jamais trouvé aucune bête si parfaite, qu'elle ait usé de quelque signe, pour faire entendre à d'autres animaux quelque chose qui n'eut point à ses passions; et il n'y a point d'homme si imparfait, qu'il n'en use ; en sorte que ceux qui sont sourds et muets, inventent des signes particuliers, par lesquels ils expriment leurs pensées. Ce qui me semble un très fort argument pour prouver que ce qui fait que les bêtes ne parlent point comme nous, est qu'elles n'ont aucune pensée, et non point que les organes leurs manquent. Et on ne peut dire qu'elles parlent entre elles mais que nous ne les entendons pas; car, comme les chiens et quelques autres animaux nous expriment leurs passions, ils nous exprimeraient aussi bien leurs pensées, s'ils en avaient. 
Je sais bien que les bêtes font beaucoup de choses mieux que nous, mais je ne m'en étonne pas ; car cela même sert à prouver qu'elles agissent naturellement et par ressort, ainsi qu'une horloge, laquelle montre bien mieux l'heure qu'il est que notre jugement ne nous l'enseigne. Et sans doute que, lorsque les hirondelles viennent au printemps, elles agissent en cela comme des horloges. Tout ce que font les mouches à miel est de même nature, et l'ordre que tiennent les grues en volant, et celui qu'observent les singes en se battant, s'il est vrai qu'ils en observent quelqu'un, et enfin l'instinct d'ensevelir leurs morts n'est pas plus étrange que celui des chiens et des chats, qui grattent la terre pour ensevelir leurs excréments, bien qu'ils ne les ensevelissent presque jamais, ce qui montre qu'ils ne le font que par instinct et sans y penser. On peut seulement dire que, bien que les bêtes ne fassent aucune action qui nous assure qu'elles pensent, toutefois, à cause que les organes de leurs corps ne sont pas fort différents des nôtres, on peut conjecturer qu'il y a quelque pensée jointe à ces organes, ainsi que nous expérimentons en nous, bien que la leur soit beaucoup moins parfaite. À quoi je n'ai rien à répondre, sinon que, si elles pensaient ainsi que nous, elles auraient une âme immortelle aussi bien que nous, ce qui n'est pas vraisemblable, à cause qu'il n'y a point de raison pour le croire de quelques animaux, sans le croire de tous, et qu'il y en a plusieurs trop imparfaits pour pouvoir croire cela d'eux, comme sont les huîtres, les éponges, etc. Mais je crains de vous importuner par ces discours, et tout le désir que j'ai est de vous témoigner que je suis etc. 
5-

\title{
Descartes à Élisabeth
}

\section{Egmond, $1^{\text {er }}$ septembre 1645.}

\author{
(AT. IV - pp. 280-287) \\ [Copie manuscrit Marburg, Staatarchiv, lettre de Descartes \\ $\left.n^{\circ} 4\right]$
}

$\underline{\text { Retour à la table des matières }}$

Madame,

Étant dernièrement incertain si Votre Altesse était à La Haye ou à Rhenen, j'adressai ma lettre par Leyde, et celle que vous m'aviez fait l'honneur de m'écrire ne me fut rendue qu'après que le messager, qui l'avait portée à Alckmar, en fut parti. Ce qui m'a empêché de pouvoir témoigner plutôt combien je suis glorieux de ce que le jugement que j'ai fait du livre ${ }^{1}$ que vous avez pris la peine de lire, n'est pas différent du vôtre et que ma façon de raisonner vous apparaît assez naturelle. Je m'assure que, si vous aviez eu le loisir de penser, autant que je l'ai fait, aux choses dont il traite, je n'en pourrais rien écrire, que vous n'eussiez mieux remarqué que moi ; mais parce que l'âge, la naissance, les occupations de V.A. ne l'ont pu permettre, peut-être que ce que j'écris pourra servir pour vous épargner un peu de temps, et que mes fautes même vous fourniront des occasions pour remarquer la vérité.

$1 \quad$ Il s'agit du De vita beata de Sénèque 
Comme, lorsque j'ai parlé d'une béatitude qui dépend entièrement de notre libre-arbitre et que tous les hommes peuvent acquérir sans aucune assistance d'ailleurs, vous remarquez fort bien qu'il y a des maladies qui, ôtant le pouvoir de raisonner, ôtent aussi celui de jouir d'une satisfaction d'esprit raisonnable ; et cela m'apprend que ce que j'avais dit généralement de tous les hommes, ne doit être entendu que de ceux qui ont l'usage libre de leur raison, et avec cela qui savent le chemin qu'il faut tenir pour parvenir à cette béatitude. Car il n'y a personne qui ne désire se rendre heureux ; mais plusieurs n'en savent pas le moyen; et souvent l'indisposition qui est dans le corps, empêche que la volonté ne soit libre. Comme il arrive aussi quand nous dormons ; car le plus grand philosophe du monde ne saurait s'empêcher d'avoir de mauvais songes, lorsque son tempérament l'y dispose. Toutefois, l'expérience fait voir que, si on a eu souvent quelque pensée, pendant qu'on a eu l'esprit en liberté, elle revient encore après, quelque indisposition qu'ait le corps ; ainsi, je puis dire que mes songes ne me représentent jamais rien de fâcheux et sans doute qu'on a grand avantage de s'être dès longtemps accoutumé à n'avoir point de tristes pensées. Mais nous ne pouvons répondre absolument de nous-mêmes, que pendant que nous sommes à nous, et c'est moins de perdre la vie que de perdre l'usage de la raison ; car, même sans les enseignements de la foi, la seule philosophie naturelle fait espérer à notre âme un état plus heureux après la mort, que celui où elle est à présent ; et elle ne lui fait rien craindre de plus fâcheux, que d'être attachée à un corps qui lui ôte entièrement sa liberté.

Pour les autres indispositions qui ne troublent pas tout à fait le sens, mais altèrent seulement les humeurs, et font qu' on se trouve extraordinairement enclin à la tristesse, à la colère ou à quelque autre passion, elles donnent sans doute de la peine, mais elles peuvent être surmontées, et même donnent matière à l'âme d'une satisfaction d'autant plus grande qu'elles ont été plus difficiles à vaincre. Et je crois aussi le semblable de tous les empêchements de dehors, comme de l'éclat d'une grande naissance, des cajoleries de la cour, des adversités de la fortune, et aussi des grandes prospérités, lesquelles ordinairement empêchent plus qu'on ne puisse jouer le rôle de Philosophe, que ne font ses disgrâces. Car, lorsqu'on a toutes choses à souhait, on s'oublie de penser à soi, et quand, par après, la fortune change, on se trouve d'autant plus surpris, qu'on s'était plus fié en elle. Enfin, on peut dire généralement qu'il n'y a aucune chose qui nous puisse entièrement ôter le moyen de nous rendre heureux, pourvu qu'elle ne trouble point notre raison; et que ce ne sont pas toujours celles qui paraissent les plus fâcheuses, qui nuisent le plus.

Mais afin de savoir exactement combien chaque chose peut contribuer à notre contentement, il faut considérer quelles sont les causes qui le produisent, 
et c'est aussi l'une des principales connaissances qui peuvent servir à faciliter l'usage de la vertu ; car toutes les actions de notre âme qui acquièrent quelque perfection, sont vertueuses, et tout notre contentement ne consiste qu'au témoignage intérieur que nous avons d'avoir quelque perfection. Ainsi nous ne saurions jamais pratique aucune vertu (c'est-à-dire ce que notre raison nous persuade que nous devons faire), que nous n'en recevions de la satisfaction et du plaisir. Mais il y a deux sortes de plaisirs : les uns qui appartiennent à l'esprit seul, et les autres qui appartiennent à l'homme, c'est-à-dire à l'esprit en tant qu'il est uni au corps ; et ces derniers, se présentant confusément à l'imagination, paraissent souvent beaucoup plus grands qu'ils ne sont, principalement avant qu' on les possède, ce qui est la source de tous les maux et de toutes les erreurs de la vie. Car, selon la règle de la raison, chaque plaisir se devrait mesurer par la grandeur de la perfection qui le produit, et c'est ainsi que nous mesurons ceux dont les causes nous sont clairement connues. Mais souvent la passion nous fait croire certaines choses beaucoup meilleures et plus désirables qu'elles ne sont ; puis, quand nous avons pris bien de la peine à les acquérir, et perdu cependant l'occasion de posséder d'autres biens plus véritables, la jouissance nous en fait connaître les défauts, et de là viennent les dédains, les regrets et les repentirs. C'est pourquoi le vrai office de la raison est d'examiner la juste valeur de tous les biens dont l'acquisition semble dépendre en quelque façon de notre conduite, afin que nous ne manquions jamais d'employer tous nos soins, à tâcher de nous procurer ceux qui sont, en effet, les plus désirables ; en quoi, si la fortune s'oppose à nos desseins et les empêche de réussir, nous aurons au moins la satisfaction de n'avoir rien perdu par notre faute et ne laissons pas de jouir de toute la béatitude naturelle dont l'acquisition aura été en notre pouvoir.

Ainsi, par exemple, la colère peut quelquefois exciter en nous des désirs de vengeance si violents qu'elle nous fera imaginer plus de plaisirs à châtier notre ennemi, qu'à conserver notre honneur ou notre vie, et nous fera exposer imprudemment l'un et l'autre pour ce sujet. Au lieu que, si la raison examine quel est le bien ou la perfection sur laquelle est fondé ce plaisir, qu'on tire de la vengeance, elle n'en trouvera aucun autre (au moins quand cette vengeance ne sert point pour empêcher qu'on ne nous offense derechef), sinon que cela nous fait imaginer que nous avons quelque supériorité et quelque avantage audessus de celui dont nous nous vengeons. Ce qui n'est souvent qu'une vaine imagination, qui ne mérite point d'être estimée à comparaison de l'honneur ou de la vie, ni même à comparaison de la satisfaction qu' on aurait de se voir maître de la colère, en s'abstenant de se venger.

Et le semblable arrive en toutes les autres passions ; car il n'y en aucune qui ne nous représente le bien auquel elle tend avec plus d'éclat qu'il n'en 
mérite, et qui ne nous fasse imaginer des plaisirs beaucoup plus grands, avant que nous les possédions, que nous les trouvons par après, quand nous les avons. Ce qui fait qu'on blâme communément la volupté, parce qu'on ne se sert de ce mot que pour signifier des plaisirs qui nous trompent souvent par leur apparence, et nous en font négliger d'autres beaucoup plus solides, mais dont l'attente ne nous touche pas tant, tels que sont ordinairement ceux de l'esprit seul. Je dis ordinairement ; car tous ceux de l'esprit ne sont pas louables, parce qu'ils peuvent être fondés sur quelque fausse opinion, comme le plaisir qu'on prend à médire, qui n'est fondé que sur ce qu'on pense devoir être d'autant plus estimé que les autres le seront moins ; et ils nous peuvent aussi tromper par leur apparence, lorsque quelque forte passion les accompagne, comme on voit en celui que donne l'ambition.

Mais la principale différence qui est entre les plaisirs du corps et ceux de l'esprit, consiste en ce que, le corps étant sujet à un changement perpétuel, et même sa conservation et son bien-être dépendant de ce changement, tous les plaisirs qui le regardent ne durent guère ; car ils procèdent que de l'acquisition de quelque chose qui est utile au corps, au moment qu'on les reçoit ; et sitôt qu'elle cesse de lui être utile, ils cessent aussi, au lieu que ceux de l'âme peuvent être immortels comme elle, pourvu qu'ils aient un fondement si solide que ni la connaissance de la vérité, ni fausse persuasion ne le détruisent.

Au reste, le vrai visage de notre raison pour la conduite de la vie ne consiste qu'à examiner et considérer sans passion la valeur de toutes les perfections, tant du corps que de l'esprit, qui peuvent être acquises par notre conduite, afin qu'étant ordinairement obligés de nous priver de quelques unes, pour avoir les autres, nous choisissions toujours les meilleures. Et parce que celles du corps sont les moindres, on peut dire généralement que, sans elles, il y a moyen de se rendre heureux. Toutefois, je ne suis point d'opinion qu'on les doive entièrement mépriser, ni même qu'on doive s'exempter d'avoir des passions ; il suffit qu'on les rende sujettes à la raison, et, lorsqu' on les a ainsi apprivoisées, elles sont quelquefois d'autant plus utiles qu'elles penchent plus vers l'excès. Je n'en aurai jamais de plus excessive, que celle qui me porte au respect et à la vénération que je vous dois et me fait être,

Madame,

de Votre Altesse

le très humble et très obéissant serviteur.

Descartes.

D’Egmond, le premier sept. 1645 
6-

\title{
Descartes à Chanut
}

\section{La Haye, 6 juin 1647.}

\author{
(AT. V - pp. 50-58) \\ [Édition Clerselier tome I, letter 36 pp.119-126]
}

$\underline{\text { Retour à la table des matières }}$

Monsieur,

Comme je passais par ici pour aller en France, j'ai appris que Monsieur Brasset qui m'avait envoyé de vos lettres à Egmont, et bien que mon voyage soit assez pressé, je me proposais de les atteindre ; mais ayant été reçues en mon logis trois heures après que j'en étais parti, on me les a incontinent renvoyées. Je les ai lues avec avidité. J'y ai trouvé de grandes preuves de votre amitié et de votre adresse. J'ai eu peur, en lisant les premières pages, où vous m'apprenez que Monsieur du Rier avait parlé à la Reine d'une de mes lettres, et qu'elle demandait de la voir. Par après, je me suis rassuré étant à l'endroit où vous écrivez qu'elle en a ouï la lecture avec quelque satisfaction; et je doute si j'ai été touché de plus d'admiration, de ce qu'elle a si facilement entendu des choses que les plus doctes estiment très obscures, et de joie, de ce qu'elles ne lui ont pas déplu. Mais mon admiration s'est redoublée, lorsque j'ai vu la force et le poids des objections que Sa Majesté a remarquées, tou- 
chant la grandeur que j'ai attribuée à l'univers. Et je souhaiterais que votre lettre m'eût trouvé en mon séjour ordinaire, parce qu'y pouvant mieux recueillir mon esprit que dans la chambre d'une hôtellerie, j' aurais peut-être pu me démêler un peu mieux d'une question si difficile et s judicieusement proposée. Je ne prétends pas toutefois que cela me serve d'excuse, et pourvu qu'il me soit permis de penser que c'est à vous seul que j'écris, afin que la vénération et le respect ne rendent point mon imagination trop confuse, je m'efforcerai ici de mettre tout ce que je puis dire touchant cette matière.

En premier lieu, je me souviens que le Cardinal de Cusa et plusieurs autres docteurs ont supposé le monde infini, sans qu'ils aient jamais été repris de l'Église pour ce sujet ; au contraire, on croit que c'est honorer Dieu, que de faire concevoir ses œuvres fort grands. Et mon opinion est moins difficile à recevoir que la leur ; parce que je ne dis pas que le monde soit infini, mais indéfini seulement. En quoi il y a une différence assez remarquable : car pour dire qu'une chose est infinie, on doit avoir quelque raison qui la fasse connaître telle, ce qu'on ne peut avoir que de Dieu seul ; mais pour dire qu'elle est indéfinie, il suffit de n'avoir point de raison par laquelle on puisse prouver qu'elle ait des bornes. Ainsi il me semble qu'on ne peut prouver, ni même concevoir, qu'il y ait des bornes en la matière dont le monde est composé. Car en examinant la nature de cette matière, je trouve qu'elle ne consiste en autre chose qu'en ce qu'elle a de l'étendue en longueur, largeur et profondeur, de façon que tout ce qui a ces trois dimensions est une partie de cette matière ; et il ne peut y avoir aucun espace entièrement vide, c'est-à-dire qui ne contienne aucune matière, à cause que nous ne saurions concevoir un tel espace, que nous ne concevions en lui ces trois dimensions, et, par conséquent, de la matière. Or, en supposant le monde fini, on imagine au-delà de ses bornes quelques espaces qui ont leurs trois dimensions, et ainsi ne sont pas purement imaginaires, comme les Philosophes les nomment, mais qui contiennent en soi de la matière, laquelle ne pouvant être ailleurs que dans le monde, fait voir que le monde s'étend au-delà des bornes qu'on avait voulu lui attribuer. N'ayant donc aucune raison pour prouver et même ne pouvant concevoir que le monde ait des bornes, je le nomme indéfini. Mais je ne puis nier pour cela qu'il en ait peut-être quelques-unes qui sont connues de Dieu, bien qu'elles me soient incompréhensibles : c'est pourquoi je ne dis pas absolument qu'il est infini.

Lorsque son étendue est considérée en cette sorte, si on la compare avec sa durée, il me semble qu'elle donne seulement occasion de penser qu'il n'y a point de temps imaginable, avant la création du monde, auquel Dieu n'eut pu le créer, s'il eût voulu ; et qu'on n'a point sujet pour cela de conclure qu'il l'a véritablement créé avant un temps indéfini, à cause que l'existence actuelle ou 
véritable que le monde a eue depuis cinq ou six mille ans n'est pas nécessairement jointe avec l'existence possible ou imaginaire qu'il a pu avoir auparavant ; ainsi que l'existence actuelle des espaces qu'on conçoit autour d'un globe (c'est-à-dire d'un monde supposé comme fini) est jointe avec l'existence actuelle de ce même globe. Outre cela, si de l'étendue indéfinie du monde on pouvait inférer l'éternité de sa durée au regard du temps passé, on la pourrait encore mieux inférer de l'éternité de la durée qu'il doit avoir à l'avenir. Car la foi nous enseigne que, bien que la terre et les cieux périront, c'est-à-dire changeront de face, toutefois le monde, c'est-à-dire la matière dont il est composé ne périra jamais ; car il paraît de ce qu'elle promet une vie éternelle à nos corps après la résurrection, et par conséquent aussi au monde dans lequel ils seront. Mais de cette durée infinie que le monde doit avoir à l'avenir, on n'infère point qu'il ait été ci-devant de toute éternité, à cause que tus les moments de sa durée sont indépendants les uns des autres.

Pour les prérogatives que la Religion a attribuées à l'homme, et qui semblent difficiles à croire, si l'étendue de l'Univers est supposée indéfinie, elles méritent quelque explication. Car, bien que nous puissions dire que toutes les choses créées sont faites pour nous, en tant que nous en pouvons tirer quelque usage, je ne sache point néanmoins que nous soyons obligés de croire que l'homme soit la fin de la Création. Mais il est dit que omnia propter (Deum) facta sunt, que c'est Dieu seul qui est la cause finale, aussi bien que la cause efficiente de l'Univers; et pour les créatures, d'autant qu'elles servent réciproquement les unes aux autres, chacune se peut attribuer cet avantage, que toutes celles qui lui servent sont faites pour elle. Il est vrai que les six jours de la Création sont tellement décrits en la Genèse, qu'il semble que l'homme en soit le principal sujet; mais on peut dire que cette histoire de la Genèse ayant été écrite pour l'homme, ce sont principalement les choses qui le regardent que le Saint Esprit a voulu spécifier, et qu'il n'y est parlé d'aucunes, qu'en tant qu'elles se rapportent à l'homme. Et à cause que les Prédicateurs, ayant soin de nous inciter à l'amour de Dieu, ont coutume de nous représenter les divers usages que nous tirons des autres créatures, et disent que Dieu les a faites pour nous, et qu'ils ne nous font point considérer les autres fins pour lesquelles on peut aussi dire qu'il les a faites, à cause que cela ne sert point à leur sujet, nous sommes fort enclins à croire qu'il ne les a faites que pour nous. Mais les Prédicateurs passent plus outre : car ils disent que chaque homme en particulier est redevable à Jésus-Christ de tout le sang qu'il a répandu en la Croix, tout de même que s'il n'était mort que pour un seul. En quoi ils disent bien la vérité ; mais comme cela n'empêche pas qu'il n'ait racheté de ce même sang un très grand nombre d'hommes, ainsi je ne vois point que le mystère de l'Incarnation et tous les autres avantages que Dieu a fait à l'homme, 
empêchent qu'il n'en puisse avoir une infinité d'autres très grands à une infinité d'autres créatures. Et bien que je n'infère point pour cela qu'il y ait des créatures intelligentes dans les étoiles ou ailleurs, je ne vois pas aussi qu'il $y$ ait aucune raison, par laquelle on puisse prouver qu'il n'y en ait point ; mais je laisse toujours indécises les questions qui sont de cette sorte, plutôt que d'en rien nier ou assurer. Il me semble qu'il ne reste plus ici autre difficulté, sinon qu'après avoir cru longtemps que l'homme a de grands avantages pardessus les autres créatures, il semble qu'on les perde tous, lorsqu'on vient à changer d'opinion. Mais je distingue entre ceux de nos biens qui peuvent devenir moindres, de ceux que d'autres en possèdent de semblables, et ceux que cela peut rendre moindres. Ainsi, un homme qui n'a que mille pistoles serait fort riche s'il n'y avait point d'autres personnes au monde qui en eussent tant; et le même serait fort pauvre, s'il n'y avait personne qui n'en eût beaucoup davantage. Et ainsi toutes les qualités louables donnent d'autant plus de gloire à ceux qui les ont, qu'elles se rencontrent en moins de personnes ; c'est pourquoi on a coutume de porter envie à la gloire et aux richesses d'autrui. Mais la vertu, la science, la santé et généralement tous les autres biens, étant considérés en eux-mêmes, sans être rapportés à la gloire, ne sont aucunement moindres en nous de ce qu'ils se trouvent aussi en beaucoup d'autres ; c'est pourquoi nous n'avons aucun sujet d'être fâchés qu'ils soient en plusieurs. Or les biens qui peuvent être en toutes les créatures intelligentes d'un monde indéfini sont de ce nombre ; ils ne rendent pas moindres ceux que nous possédons. Au contraire, lorsque nous aimons Dieu, et que par lui nous nous joignons de volonté avec toutes les choses qu'il a créées, d'autant que nous les concevons plus grandes, plus nobles, plus parfaites, d'autant nous estimons nous aussi davantage, à cause que nous sommes des parties d'un tout plus accompli ; et d'autant avons nous plus de sujet de louer Dieu, à cause de l'immensité de ses œuvres. Lorsque l'Écriture sainte parle en divers endroits de la multitude innombrable des Anges, elle confirme entièrement cette opinion : car nous jugeons que les moindres Anges sont incomparablement plus parfaits que les hommes. Et les Astronomes, qui, en mesurant la grandeur des étoiles, les trouvent beaucoup plus grandes que la terre, la confirment aussi : car si, de l'étendue indéfinie du monde, on infère qu'il doit y avoir des habitants ailleurs qu'en la terre, on le peut inférer aussi de l'étendue que tous les Astronomes lui attribuent; à cause qu'il n'y en a aucun qui ne juge que la terre est plus petite au regard de tout le Ciel, que n'est un grain de sable au regard d'une montagne.

Je passe maintenant à votre question, touchant les causes qui nous incitent souvent à aimer une personne plutôt qu'une autre, avant que nous en connaissions le mérite ; et j'en remarque deux, qui sont, l'une dans l'esprit, l'autre 
dans le corps. Mais pour celle qui n'est que dans l'esprit, elle présuppose tant de choses touchant la nature de nos âmes, que je n'oserais en entreprendre de les déduire dans une lettre. Je parlerai seulement de celle du corps. Elle consiste dans la disposition des parties de notre cerveau, soit que cette disposition ait été mise en lui par les objets des sens, soit par quelque autre cause. Car les objets qui touchent nos sens meuvent par l'entremise des nerfs quelques parties de notre cerveau, et y font comme certains plis, qui se défont lorsque l'objet cesse d'agir ; mais la partie où ils ont été faits demeure par après disposée à être pliée derechef de la même façon par un autre objet qui ressemble en quelque chose au précédent, encore qu'il ne lui ressemble pas du tout. Par exemple, lorsque j'étais enfant, j'aimais une fille de mon âge, qui était un peu louche ; au moyen de quoi, l'impression qui se faisait par la vue en mon cerveau, quand je regardais ses yeux égarés, se joignait tellement à celle qui s'y faisait aussi pour émouvoir en moi la passion de l'amour, que longtemps après, en voyant des personnes louches, je me sentais plus enclin à les aimer qu'à en aimer d'autres, pour cela seul qu'elles avaient ce défaut; et je ne savais pas néanmoins que ce fût pour cela. Au contraire, depuis que j'y ai fait réflexion, et j'ai reconnu que c'était un défaut, je n'en ai plus été ému. Ainsi, lorsque nous sommes portés à aimer quelqu'un, sans que nous en sachions la cause, nous pouvons croire que cela vient de ce qu'il y a quelque chose en lui de semblable à ce qui a été dans un autre objet que nous avons aimé auparavant, encore que nous ne sachions pas ce que c'est. Et bien que ce soit plus ordinairement une perfection qu'un défaut qui nous attire ainsi à l'amour ; toutefois, à cause que ce peut être quelquefois un défaut, comme en l'exemple que $\mathrm{j}$ 'ai apporté, un homme sage ne se doit pas laisser entièrement aller à cette passion, avant que d'avoir considéré le mérite de la personne pour laquelle nous nous sentons émus. Mais à cause que nous ne pouvons pas aimer également tous ceux en qui nous remarquons des mérites égaux, je crois que nous sommes seulement obligés de les estimer également; et que, le principal bien de la vie étant d'avoir de l'amitié pour quelques-uns, nous avons raison de préférer ceux à qui nos inclinations secrètes nous joignent, pourvu que nous remarquions aussi en eux du mérite. Outre que, lorsque ces inclinations secrètes ont leur cause en l'esprit, et non dans le corps, je crois qu'elles doivent toujours être suivies; et la marque principale qui les fait connaître, est que celles qui viennent de l'esprit sont réciproques, ce qui n'arrive pas souvent aux autres. Mais les preuves que j'ai de votre affection m'assurent si fort que l'inclination que j'ai pour vous est réciproque, qu'il faudrait que je fusse entièrement ingrat et que je manquasse à toutes les règles que je crois devoir être observées en l'amitié, si je n'étais pas, avec beaucoup de zèle, etc.

A La Haye, le 6 juin 1647. 
7-

\title{
Descartes à Gibieuf
}

\section{9 janvier 1642}

\author{
(AT. II - pp. 472-480) \\ [Version française de Clerselier, tome 1, lettre 105, p.479- \\ 484]
}

$\underline{\text { Retour à la table des matières }}$

Monsieur et Révérend Père,

J'ai toujours assez éprouvé combien vous favorisiez le désir que j'ai de faire quelque progrès en la recherche de la vérité et le témoignage que vous m'en rendez encore par lettres m'oblige extrêmement. Je suis aussi très obligé au Révérend Père de la Barde, pour avoir pris la peine d'examiner mes pensées de métaphysique, et m'avoir fait la faveur de les défendre contre ceux qui m'accusaient de mettre tout en doute. Il a très parfaitement pris mon intention, et si j'avais plusieurs protecteurs tels que vous et lui, je ne douterais point que mon parti ne se rendit bientôt le plus fort; mais quoi que je n'en aie que fort peu, je ne laisse pas d'avoir beaucoup de satisfaction de ce que ce sont les plus grands hommes et les meilleurs esprits qui goûtent et favorisent le plus mes opinions. Je me laisse aisément persuader que, si le Révérend Père de Gondran eût vécu, il en aurait été des principaux ; et bien qu'il n'y ait pas longtemps que Monsieur Arnaut est docteur, je ne laisse pas d'estimer plus son jugement qu'une moitié des anciens. Mon espérance n'a point été 
d'obtenir leur approbation en corps; j'ai trop bien su et prédit, il y a longtemps, que mes pensées ne seraient pas au goût de la multitude, et qu'où la pluralité des voix aurait lieu, elles seraient aisément condamnées. Je n'ai pas aussi désiré celle des particuliers, à cause que je serais marri qu'ils fissent rien à mon sujet, qui peut être désagréable aux yeux de leurs confrères, et aussi qu'on a coutume de l'obtenir si facilement pour tous les livres qui ne sont plus hérétiques que le mien, que j' ai cru que la cause pour laquelle on pourrait juger que je ne l'ai pas, ne me serait point désavantageuse. Mais cela ne m'a pas empêché d'offrir mes Méditations à votre Faculté, afin de les faire d'autant mieux examiner, et que, si ceux d'un Corps si célèbre ne trouvaient point de justes raisons pour les reprendre, cela me peut assurer des vérités qu'elles contiennent.

Pour ce qui est du principe par lequel il me semble connaître que l'idée que j'ai de quelque chose, non redditur a me inadaequata per abstractionem intellectus, je ne le tire que de ma propre pensée ou conscience. Car étant assuré que je ne puis avoir aucune connaissance de ce qui est hors de moi que par l'entremise des idées que j'en ai eu en moi, je me garde bien de rapporter mes jugements immédiatement aux choses, et de leur rien attribuer de positif, que je ne l'aperçoive auparavant en leurs idées ; mais je crois aussi que tout ce qui se trouve en ces idées, est nécessairement dans les choses. Ainsi, pour savoir si mon idée n'est point rendue non complète ou inadaequata, par quelque abstraction de mon esprit, j'examine seulement si je ne l'ai point tirée, non de quelque chose hors de moi qui soit plus complète, mais de quelque autre idée plus ample ou plus complète que j'ai en moi, et ce per abstractionem intellectus, c'est-à-dire en détournant ma pensée d'une partie de ce qui est compris en cette idée plus ample, pour l'appliquer d'autant mieux et me rendre plus d'autant plus attentif à l'autre partie. Ainsi, lorsque je considère une figure sans penser à la substance ni à l'extension dont elle est figure, je fais une abstraction d'esprit que je puis aisément reconnaître par après, en examinant si je n'ai point tiré cette idée que j'ai, de la figure seule, hors de quelque autre idée plus ample que j'ai aussi en moi, à qui elle soit tellement jointe que, bien qu' on puisse penser à l'une sans avoir aucune attention à l'autre, on ne puisse toutefois la nier de cette autre lorsqu' on pense à toutes deux. Car je vois clairement que l'idée de figure est ainsi jointe à l'idée de l'extension et de la substance, vu qu'il est impossible que je conçoive une figure en niant qu'elle ait une extension, ni une extension en niant qu'elle soit l'extension d'une substance. Mais l'idée d'une substance étendue et figurée est complète, à cause que je la puis concevoir toute seule, et nier d'elle toutes les autres choses dont j'ai des idées. Or, il est, ce me semble, fort clair que l'idée que j'ai d'une substance qui pense, est complète en cette façon, et que je n'ai aucune autre idée qui la précède en mon esprit, et qui lui 
soit tellement jointe, que je ne les puisse bien concevoir en les niant l'une et l'autre ; car s'il y en avait quelqu'une en moi qui fût telle, je devrais nécessairement la connaître. On dira peut-être que la difficulté demeure encore, à cause que, bien que je conçoive l'âme et le corps comme deux substances que je puis concevoir l'une sans l'autre, et même en niant l'une de l'autre, je ne suis pas toutefois assuré qu'elles sont telles que je les conçois. Mais il en faut revenir à la règle ci-devant posée, à savoir que nous ne pouvons avoir aucune connaissance des choses, que par les idées que nous en concevons ; et que, par conséquent, nous n'en devons juger que suivant ces idées, et même nous devons penser que tout ce qui répugne à ces idées est absolument impossible, et implique contradiction. Ainsi nous n'avons aucune raison pour assurer qu'il n'y a point de montagne sans vallée, sinon que nous voyons que leurs idées ne peuvent être complètes quand nous les considérons l'une sans l'autre, bien que nous puissions, par abstraction, avoir l'idée d'une montagne, ou d'un lieu qui va en montant de bas en haut, sans considérer qu'on peut aussi descendre par le même de haut en bas. Ainsi, nous pouvons dire qu'il implique contradiction, qu'il y ait des atomes ou des parties de matière qui aient l'extension et toutefois soient indivisibles, à cause qu'on ne peut avoir l'idée d'une chose étendue qu'on puisse avoir aussi celle de sa moitié, ou de son tiers, ni, par conséquent, sans qu' on la conçoive divisible en 2 ou en 3. Car, de cela seul que je considère les deux moitiés d'une partie de matière, tant petite qu'elle puisse être, comme deux substances complètes, \& quarum ideae non redduntur a me inadequatae per abstractionem intellectus, je conclus certainement qu'elles sont réellement divisibles. Et si on me disait que, nonobstant que je les puisse concevoir, je ne sais pas pour cela si Dieu ne les a point unies ou jointes ensemble d'un lien si étroit qu'elles soient entièrement inséparables, et ainsi que je n'ai pas de raison de le nier; je répondrais que, de quelque lien qu'il puisse les avoir jointes, je suis aussi assuré qu'il peut aussi les disjoindre de façon qu'absolument parlant, j'ai raison de les nommer divisibles, puisqu'il m'a donné la faculté de les concevoir comme telles. Et je dis tout le même de l'âme et du corps, et généralement de toutes les choses dont nous avons des idées diverses et complètes, à savoir qu'il n'implique aucune contradiction qu'elles soient inséparables. Mais je ne nie pas pour autant qu'il ne puisse y avoir dans l'âme ou dans le corps plusieurs propriétés dont je n'ai aucunes idées ; je nie seulement qu'il y en ait aucune qui répugne aux idées que j'en ai et, entre autres, à celle que j'ai de leur distinction ; car autrement Dieu serait trompeur, et nous n'aurions aucune règle pour assurer de la vérité.

La raison pour laquelle je crois que l'âme pense toujours, est la même qui me fait croire que la lumière luit toujours, bien qu'il n'y ait point d'yeux qui la regardent ; que la chaleur est toujours chaude, bien qu'on ne s'y chauffe 
point; que le corps ou la substance étendue a toujours de l'extension; et généralement que ce qui constitue la nature d'une chose est toujours en elle pendant qu'elle existe ; en sorte qu'il me serait plus aisé de croire que l'âme cesserait d'exister, quand on dit qu'elle cesse de penser, que non pas de concevoir qu'elle fut sans pensée. Et je ne vois ici aucune difficulté, sinon qu' on juge superflu de croire qu'elle pense lorsqu'il ne nous en demeure aucun souvenir par après. Mais si on considère que nous avons toutes les nuits mille pensées, et même en veillant que nous en avons eu mille depuis une heure, dont il ne nous reste plus aucune trace en la mémoire, et dont nous ne voyons pas mieux l'utilité, que celles que nous pouvons avoir eues avant que de naître, on aura bien moins de peine à se le persuader qu'à juger qu' une substance dont la nature est de penser, puisse exister, et toutefois ne penser point.

Je ne vois aussi aucune difficulté à entendre que les facultés d'imaginer et de sentir appartiennent à l'âme, à cause que ce sont des espèces de pensées ; et néanmoins n'appartiennent qu'à l'âme en tant qu'elle est jointe au corps, à cause que ce sont des sortes de pensées, sans lesquelles on peut concevoir l'âme toute pure.

Pour ce qui est des animaux, nous remarquons bien en eux des mouvements semblables à ceux qui suivent de nos imaginations ou sentiments, mais non pas pour cela des imaginations ou sentiments. Et, au contraire, ces mêmes mouvements se pouvant aussi faire sans imagination, nous avons des raisons qui prouvent qu'ils se font ainsi en eux, comme j'espère faire voir clairement, en décrivant par le menu toute l'architecture de leurs membres et les causes de leurs mouvements.

Mais je crains que je ne vous aie déjà ennuyé par la longueur de cette lettre; je me tiendrai très heureux si vous me continuez l'honneur de votre bienfaisance et la faveur de votre protection, comme à celui qui est, etc.

Fin de la lettre. 
8-

\section{Descartes à ???}

\section{Date ???}

(AT. II - pp. 472-480)

[Version française de Clerselier, tome 1, lettre 105, p.479484]

$\underline{\text { Retour à la table des matières }}$ 